\title{
Resistivity scaling and critical dynamics of fully frustrated Josephson-junction arrays with on-site dissipation
}

\author{
Enzo Granato \\ Laboratório Associado de Sensores e Materiais, Instituto Nacional de Pesquisas Espaciais, 12201-190 São José dos Campos, \\ São Paulo, Brazil \\ Daniel Domínguez \\ Centro Atómico Bariloche and Instituto Balseiro, 8400 San Carlos de Bariloche, Rio Negro, Argentina \\ (Received 25 May 2004; revised manuscript received 20 December 2004; published 31 March 2005)
}

\begin{abstract}
We study the scaling behavior and critical dynamics of the resistive transition in Josephson-junction arrays, at $f=1 / 2$ flux quantum per plaquette, by numerical simulation of an on-site dissipation model for the dynamics. The results are compared with recent simulations using the resistively shunted-junction model. For both models, we find that the resistivity scaling and critical dynamics of the phases are well described by the same critical temperature as for the chiral (vortex-lattice) transition, with a power-law divergent correlation length. The behavior is consistent with the single transition scenario, where phase and chiral variables order at the same temperature, but with different dynamic exponents $z$ for phase coherence and chiral order.
\end{abstract}

DOI: 10.1103/PhysRevB.71.094521 PACS number(s): 74.50.+r, 64.60.Cn, 64.60.Ht, 74.60.Ge

\section{INTRODUCTION}

There has been considerable interest, both experimentally and theoretically, in phase transitions of two-dimensional Josephson-junction arrays (JJA). ${ }^{1-41}$ Such arrays can be artificially fabricated as a lattice of superconducting grains connected by an insulator or a normal metal ${ }^{1-6}$ and are also closely related to superconducting wire networks. ${ }^{7,8}$ Experimentally, the resistive transition has been the one most extensively studied, ${ }^{2-4,6-8}$ while theoretically several studies of $X Y$ models, ${ }^{9-41}$ which describe the ideal JJA, have been done. A significant understanding of these systems has already been achieved by comparing the results of experiments with the theoretically predicted equilibrium critical behavior, with and without an applied magnetic field. However, to a large extent, dynamical critical behavior remains much less understood, particularly in the presence of a magnetic field, where frustration effects may introduce additional elementary excitations relevant for the static and dynamic critical behavior. It is well known that while static critical phenomena depend on the spatial dimensionality as well as on the symmetry of the order parameter, the dynamic universality class of the phase transition will depend upon additional properties which do not affect the statics as, for example, conservation laws for the order parameter. ${ }^{42}$ Thus, testing the universality hypothesis of dynamical critical behavior requires the study of specific dynamical models. For JJA, the physically relevant dynamical model for the phase dynamics cannot be unambiguously identified ${ }^{5,9-12}$ and should depend on the particular coupling mechanism between the superconducting elements of the array. It is expected that, at least for an array of ideal tunnel junctions, the resistively shuntedjunction (RSJ) model of current flow between superconducting grains would be a more physical representation of the system. ${ }^{13}$ This model assumes that energy dissipation occurs only through the junctions and imposes current conservation at each site of the array. On the other hand, for wire networks or arrays of proximity-effect junctions, local dissipation at the sites of the array should also be allowed leading to a model with on-site dissipation for the dynamics.

In experimental investigations of JJA, the resistive transition is usually identified from the behavior of the currentvoltage $(I-V)$ characteristics near the critical temperature. The divergent correlation length determines both the linear and nonlinear resistivity sufficiently close to the transition. To interpret the data and determine the underlying equilibrium transition, the scaling theory of Fisher et al. ${ }^{43}$ has been widely used. For JJA at zero magnetic field, which is isomorphic to the standard $X Y$ model, the resistive transition is in the Kosterlitz-Thouless (KT) universality class, ${ }^{1,13,14}$ where the correlation length diverges exponentially near the critical temperature. Studies of the critical dynamical behavior using Monte Carlo (MC) dynamics ${ }^{15}$ and RSJ or on-site dissipation dynamics, ${ }^{11,12}$ find a behavior consistent with the dynamical theory of the KT transition. The exponent of the currentvoltage relation, $V-I^{a}$, at the transition, assuming the universal value $a=z+1=3$, corresponds to a dynamic exponent $z$ $=2$ in the resistivity scaling theory. ${ }^{43}$

However, in frustrated Josephson-junction arrays (FJJA), corresponding to $f=1 / 2$ flux quantum per plaquette, besides the phase variables, the vortex-lattice induced by the external field introduces an additional discrete (Ising-like) order parameter, the chirality, ${ }^{16}$ which measures the direction of local current circulation in the array. The ground state consists of a pinned commensurate vortex-lattice corresponding to an antiferromagnetic arrangement of chiralities and vortex-lattice melting corresponds to the chiral order-disorder transition. As a consequence, two distinct scenarios for the occurrence of phase transitions as a function of temperature have been proposed by Teitel and Jayaprakash (Ref. 16): separated chiral and phase-coherence transitions or a single transition where phase and chirality order at the same temperature. In the former scenario, the phase transitions should be in the $\mathrm{KT}$ and Ising universality classes, respectively, while in the 
later scenario the critical behavior should be a superposition of KT and Ising critical behavior at the same critical temperature, if the coupling between phase and chiral variables are irrelevant at criticality (decoupled single transition), otherwise critical behavior (coupled single transition) should occur with phase coherence and chiral order showing critical behavior different from the KT and Ising universality classes. These possible scenarios are supported, for example, by renormalization-group studies based on the GinzburgLandau expansion of the frustrated $X Y$ model (FXY) ${ }^{17}$ which also shows that the universality class of these transitions can be described by the $X Y$-Ising model. ${ }^{18}$ It appears that the current predominant point of view is that the separated transition scenario is realized with a KT transition occurring below the chiral transition. Recently, this scenario has received further support from appealing arguments by Korshunov, ${ }^{28}$ based on chiral domain wall fluctuations and vortex unbinding, which provides a mechanism for the separation of the two transitions in this order. Also, there are significant numerical evidences from equilibrium calculations which favor this scenario. However, the coupled single transition scenario has also received some support from different calculations of the chiral critical exponents and central charge from finitesize scaling which show results different from the pure Ising values, but several of these studies do not verify if the transition temperature for phase-coherence coincides with the chiral transition temperature. On the other hand, the numerical evidence for the separated transitions scenario finds that the phase-coherence transition ${ }^{24}$ is consistent with KT behavior but the critical exponents found for the chiral transition by finite-size scaling do not show the expected pure Ising behavior according to Ref. 23. However, it has been found by Olsson ${ }^{24}$ that the value of the thermal critical exponent is consistent with the pure Ising value depending on the temperature region in which a fit is made. Therefore, the deviations of the exponents from pure Ising values cannot be regarded as an unambiguous evidence for non-Ising critical behavior. The separated transition scenario also relies on the assumption that the phase-coherence transition is pure KT and therefore uses some of the predicted behavior from the KT theory, like the helicity modulus jump or exponentially divergent correlation length, to locate this critical temperature. If the helicity modulus jump is actually larger than the universal value then the procedure of locating the critical temperature from the jump ${ }^{24}$ can only overestimate the critical temperature. Although this assumption is consistent with a phase-coherence critical temperature below the chiral transition, such a procedure could result in an underestimate of the phase-coherence critical temperature if the transitions coincide or the chiral transition occurs below the phasecoherence transition. In fact, it has been shown that if one enlarges the parameter space of the FXY model ${ }^{29}$ by considering a model where every other column in the square lattice has coupling constants which differ from the others by a constant ratio $\rho$, the chiral transition occurs below the phasecoherence transition $^{30}$ if $\rho$ is sufficiently different from 1 . It is then found that there is a singular contribution to the temperature dependence of the helicity modulus near the chiral transition $^{29}$ determined by the chiral critical exponents. For the standard FXY model, obtained when $\rho \rightarrow 1$, such singular contribution will remain if the transition is single and therefore it can affect the helicity modulus behavior near the transition.

In any case, independently of the scenario interpretation, several numerical calculations using quite different methods agree $^{20,23,24,31,32}$ with the earlier estimate of the chiral transition temperature ${ }^{19}$ at $T_{c h}=0.455$ within a $0.8 \%$ error bar. On the other hand, for the phase-coherence transition, it is clear that it would be more satisfactorily if it could be determined by methods which do no rely on assumptions of KT behavior.

These different phase-transition scenarios have important consequences for the resistive behavior of the FJJA. Since the resistive transition corresponds to the onset of phase coherence, they imply quite distinct behavior. In the separated transition scenario or single but decoupled scenario, the resistive behavior should be described by the KT universality class. On the other hand, in the single coupled scenario, where the critical dynamics involve strongly coupled phase and chiral variables, the resistive behavior should be significantly different. In principle, such behavior can be detected experimentally.

Measurements of current-voltage curves in FJJA were fitted assuming pure KT behavior, ${ }^{2,3}$ but either an unexpectedly low value of the transition temperature (compared with theoretical expectations) was obtained in one case $\mathrm{e}^{2}$ or the $I-V$ exponent at the transition was $a<3$ in the other case. ${ }^{3}$ More recently, the current-voltage curves in $\mathrm{JJA}^{6}$ and in superconducting networks ${ }^{7,8}$ were found to be better described by a power-law correlation length. However, very different values of the critical exponents $z, \nu$ were obtained in each case.

Earlier numerical studies of the $I-V$ characteristics for FJJA, obtained with RSJ dynamics ${ }^{33,34}$ or MC dynamics, ${ }^{35}$ were performed for small system sizes $(L \leqslant 16)$. In particular, the studies with RSJ dynamics used free boundary conditions to impose a driving current. This leads to significant additional dissipation due to boundary effects, ${ }^{36}$ specially in small system sizes. Other works have studied the short-time dynamics of chirality, ${ }^{31}$ and the nonequilibrium transitions at large currents. ${ }^{37}$

Recently, ${ }^{38}$ we have studied the critical dynamics and resistivity scaling in FJJA by numerical simulation of the RSJ dynamics with periodic (fluctuating twist) boundary conditions including much large systems sizes. It was found that the current-voltage scaling is consistent with the singletransition scenario. The scaling behavior is well described by a resistive transition occurring at a critical temperature corresponding to the chiral transition, with a power-law divergent correlation length, but with two different dynamic exponents, $z_{p h} \sim 1$ and $z_{c h} \sim 2$, for phase and chiral variables, respectively. This result implies that, at the transition, the exponent of the $I-V$ power-law, $V-I^{a}$, is $a=z_{p h}+1 \approx 2$ rather than $a=3$ as for the unfrustrated case. In view of the possible dependence of the dynamic behavior on the particular RSJ dynamics used in these simulations, it should also be of interest to study the resistive behavior with an on-site dissipation model for the dynamics. Results for this dynamical model should be particularly relevant for frustrated wire networks ${ }^{8}$ or proximity-effect junctions.

In this work we study the resistivity scaling and critical dynamics of a frustrated Josephson-junction array, defined on 
square lattice, at $f=1 / 2$ flux quantum per plaquette, by numerical simulations of an on-site dissipation model for the array dynamics. Using a dynamic scaling analysis, we find that the resistivity behavior and critical dynamics are well described by the critical temperature corresponding to the chiral (vortex-lattice) transition with a correlation length that diverges as a power law. Two dynamic exponents, $z_{p h} \sim 1.5$ and $z_{c h} \sim 2.5$, are found for phase coherence and chiral order, respectively. Consequently, at the transition, the exponent of the current-voltage power-law, $V-I^{a}$, is $a=z_{p h}+1 \approx 2.5$ rather than $a=3$ as for the unfrustrated case. This is the same behavior we have found recently for the RSJ model ${ }^{38}$ but with different values for the dynamic exponents $\left[z_{p h}\right.$ $\sim 0.9(1)$ and $\left.z_{c h} \sim 2.1\right]$. Including on-site dissipation in the dynamical model could be a more realistic description of wire networks than the RSJ model. Indeed, resistivity scaling of experimental data on wire networks ${ }^{8}$ find $z \sim 2$, which is consistent with our estimate $z_{p h}$ within the experimental errors, and also shows that the resistivity scaling is well described by a power-law correlation length as found in our simulations.

\section{MODEL AND SIMULATION}

The Hamiltonian of a square two-dimensional array under a magnetic field is given by

$$
\mathcal{H}=-E_{J} \sum_{\mathbf{r}, \mu} \cos \left(\theta_{\mathbf{r}+\mu}-\theta_{\mathbf{r}}-A_{\mathbf{r}, \mu}\right),
$$

where $\theta_{\mathbf{r}}$ is the superconducting phase of the grain at site $\mathbf{r}$ $=\left(n_{x} a, n_{y} a\right)$ with $n_{x}, n_{y}$ integers, and $a$ the lattice constant, and $\mu=\mathbf{x}, \mathbf{y}$ with $\mathbf{x}=(a, 0), \mathbf{y}=(0, a)$, and $E_{J}=I_{0} \hbar / 2 e$ the Josephson energy. The magnetic field introduces frustration through the vector potential integral

$$
A_{\mathbf{r}, \mu}=\frac{2 \pi}{\Phi_{0}} \int_{\mathbf{r}}^{\mathbf{r}+\mu} \mathbf{A} \cdot d \mathbf{l},
$$

which satisfies

$$
\begin{aligned}
\Delta_{\mu} \times A_{\mathbf{r}, \mu} & =A_{\mathbf{r}, x}-A_{\mathbf{n}+\mathbf{y}, x}+A_{\mathbf{r}+\mathbf{x}, y}-A_{\mathbf{r}, y} \\
& =2 \pi f,
\end{aligned}
$$

with $f=H a^{2} / \Phi_{0}$, where $H$ is the applied magnetic field and $\Phi_{0}=h / 2 e$ is the quantum of flux. The fully frustrated case corresponds to half quantum of flux per plaquette, $f=1 / 2$.

The simulations are performed with the same "fluctuating twist" boundary conditions as used, for example, in Refs. 11, 39, and 40. This consists of considering periodic boundary conditions for the supercurrents in the $\mu$ direction while adding a fluctuating twist $\alpha_{\mu}$ to the gauge invariant phase in the $\mu$ direction. In this case the gauge invariant phase difference is modified to

$$
\theta_{\mathbf{r}, \mu}=\theta_{\mathbf{r}+\mu}-\theta_{\mathbf{r}}-A_{\mathbf{r}, \mu}+\alpha_{\mu} .
$$

For the vector potential we choose the Landau gauge

$$
A_{\mathbf{r}, x}=-2 \pi f n_{y},
$$

$$
A_{\mathbf{r}, y}=0 \text {. }
$$

In this gauge, the boundary condition for the phases in a system of size $L \times L$ is given by

$$
\begin{gathered}
\theta\left(n_{x}+L, n_{y}\right)=\theta\left(n_{x}, n_{y}\right), \\
\theta\left(n_{x}, n_{y}+L\right)=\theta\left(n_{x}, n_{y}\right)-2 \pi f L n_{x} .
\end{gathered}
$$

For $f=1 / 2$ and $L$ even, the second condition is irrelevant, but not for general frustration $f$. In the presence of an external current $I_{\text {ext }}^{\mu}$ in the $\mu$ direction, one has to add the term $-(\hbar / 2 e) L^{2} I_{\text {ext }}^{\mu} \alpha_{\mu}$ in the Hamiltonian of Eq. (1), which couples the current with the global phase difference per row, $L \alpha_{\mu}$, introduced by the fluctuating twist. Therefore, the Hamiltonian of a frustrated square array with fluctuating twist boundary conditions and an external current is

$$
\mathcal{H}=-E_{J} \sum_{\mathbf{r}, \mu} \cos \left(\theta_{\mathbf{r}+\mu}-\theta_{\mathbf{r}}-A_{\mathbf{r}, \mu}+\alpha_{\mu}\right)-\frac{\hbar}{2 e} L^{2} \sum_{\mu} I_{\mathrm{ext}}^{\mu} \alpha_{\mu} .
$$

We define the on-site dissipation dynamics by considering the local Langevin equations for the fluctuating variables $\theta_{\mathbf{r}}$ and $\alpha_{\mu}$ :

$$
\begin{gathered}
\frac{d \theta_{\mathbf{r}}}{d t}=-\Gamma_{\theta} \frac{\delta \mathcal{H}}{\delta \theta_{\mathbf{r}}}+\eta_{\mathbf{r}}(t), \\
\frac{d \alpha_{\mu}}{d t}=-\Gamma_{\alpha} \frac{\delta \mathcal{H}}{\delta \alpha_{\mu}}+\eta_{\mu}(t),
\end{gathered}
$$

where $\Gamma_{\theta}, \Gamma_{\alpha}$ are dissipation parameters, and the noise terms have zero average and correlations

$$
\begin{gathered}
\left\langle\eta_{\mathbf{r}}(t) \eta_{\mathbf{r}^{\prime}}\left(t^{\prime}\right)\right\rangle=2 k_{B} T \Gamma_{\theta} \delta_{\mathbf{r}, \mathbf{r}^{\prime}} \delta\left(t-t^{\prime}\right), \\
\left\langle\eta_{\mu}(t) \eta_{\mu^{\prime}}\left(t^{\prime}\right)\right\rangle=2 k_{B} T \Gamma_{\alpha} \delta_{\mu, \mu^{\prime}} \delta\left(t-t^{\prime}\right) .
\end{gathered}
$$

The dissipation constant $\Gamma_{\alpha}$ should be proportional to $L^{-2}$ in order to be an intensive quantity. A convenient choice is

$$
\Gamma_{\alpha}=\frac{\Gamma_{\theta}}{L^{2}}
$$

(in general it can be $\Gamma_{\alpha}=\beta \Gamma_{\theta} / L^{2}$, here we choose $\beta=1$ to be consistent with Ref. 12).

Dimensionless quantities are used with time in units of $\tau=2 e / \hbar \Gamma_{\theta} I_{0}$, currents in units of $I_{0}$, voltages in units of $(\hbar / 2 e)^{2} \Gamma_{\theta} I_{0}$ and temperature in units of $\hbar I_{0} / 2 e k_{B}$. A total current $I$ is imposed uniformly in the array in the $\mathbf{y}$ direction with current density $J=I / L$, where $L$ is the system size and the average electric field $E$ is obtained from the voltage $V$ across the system as $E=V / L=(\hbar / 2 e)\left\langle d \alpha_{y} / d t\right\rangle$, where $\alpha_{y} L$ is the global phase difference or twist in the $\mathbf{y}$ direction. With all this considerations, the dimensionless equations of motion are then

$$
\frac{d \theta_{\mathbf{r}}}{d t}=-\Delta_{\mu} \cdot S_{\mathbf{r}, \mu}+\eta_{\mathbf{r}}(t)
$$




$$
\frac{d \alpha_{\mu}}{d t}=-\frac{1}{L^{2}} \sum_{\mathbf{r}} S_{\mathbf{r}, \mu}+I \delta_{\mu, y}+\eta_{\mu}(t)
$$

where the supercurrent is defined by

$$
S_{\mathbf{r}, \mu}=\sin \left(\theta_{\mathbf{r}+\mu}-\theta_{\mathbf{r}}-A_{\mathbf{r}, \mu}+\alpha_{\mu}\right),
$$

and the discrete divergence operator is defined as

$$
\Delta_{\mu} \cdot S_{\mathbf{r}, \mu}=\sum_{\mu=x, y} S_{\mathbf{r}, \mu}-S_{\mathbf{r}-\mu, \mu}
$$

Finally, the now dimensionless noise variables $\eta_{\mathbf{r}}(t)$ have correlations

$$
\begin{aligned}
\left\langle\eta_{\mathbf{r}}(t) \eta_{\mathbf{r}^{\prime}}\left(t^{\prime}\right)\right\rangle & =2 T \delta_{\mathbf{r}, \mathbf{r}^{\prime}} \delta(t-t), \\
\left\langle\eta_{\mu}(t) \eta_{\mu^{\prime}}\left(t^{\prime}\right)\right\rangle & =\frac{2 T}{L^{2}} \delta_{\mu, \mu^{\prime}} \delta(t-t) .
\end{aligned}
$$

The set of Eqs. (8) and (9) describe the dynamics of JJA with "on-site dissipation" in contrast to the RSJ dynamics which only considers dissipation through the junctions. ${ }^{13}$ The on-site dissipation dynamical model has been studied previously $9,11,12,40$ for the unfrustrated $(f=0)$ case and compared with the RSJ dynamics. Their main difference is that while the on-site dynamics corresponds to a local damping the RSJ dynamics corresponds to a nonlocal damping. ${ }^{9,13} \mathrm{~A}$ physical interpretation of the on-site dynamics for JJA in terms of currents and voltages has also been discussed previously. ${ }^{9,11,12,40}$ Its main features are summarized in the following. (i) It takes into account normal current flow between each superconducting node and the substrate, which leads to a current leakage through a resistance to the ground $R_{0}$. (ii) It neglects the quasiparticle normal current of each junction, which is associated with a shunt resistante $R_{s}$. This means taking $R_{s} \rightarrow \infty$, or actually assuming $R_{s} \gg R_{0}$ for the array. The assumptions (i) and (ii) lead to Eq. (8) for $\theta_{\mathbf{r}}$, which corresponds to the conservation of supercurrents at each node plus a leakage of normal current to the substrate. In this case we get

$$
\Gamma_{\theta}=(2 e / \hbar)^{2} R_{0} .
$$

However, if one considers Eq. (8) alone for the calculation of current-voltage curves with open boundary conditions it is found that an applied external current leads to dissipation only at the boundaries were current is injected (extracted), since normal current will flow directly from the first (last) row of junctions to the substrate through $R_{0}{ }^{44}$ Strictly periodic boundary conditions are not possible to be implemented in a consistent way. (iii) In order to correctly model currentvoltage curves and to be able to implement fluctuating twist periodic boundary conditions, one has to $\operatorname{add}^{11,40}$ a global normal current channel in parallel to the whole array, with a "global resistance" $R_{\text {global }}$, such that in the normal state the total resistance of the array will be given by $R_{\text {global }}$. Then total conservation of current leads to Eq. (9) which represents a parallel circuit of the average supercurrent in the array and the global normal current. In the approach of Refs. 11,12 , and $40 R_{\text {global }}=R_{0}$ is assumed, and therefore this leads to the choice $\Gamma_{\alpha}=(2 e / \hbar)^{2} R_{0} / L^{2}$.
The earlier assumptions (i)-(iii) for the on-site dynamics give a consistent interpretation of calculations of the currentvoltage response and phase dynamics, but correspond to a model system rather than to a particular JJA available experimentally. In realistic JJA the normal currents in the junctions can not be neglected since usually $R_{S} \ll R_{0}$, and therefore the RSJ model can be a good representation of the JJA. A possible realization of the dynamics of Eqs. (8) and (9) could be achieved experimentally if one adds on purpose a resistor in parallel to the whole array such that it has a resistance $R_{\text {global }} \ll R_{s}$, in which case normal currents will mainly go through $R_{\text {global }}$ and reduce the weight of the normal quasiparticle currents of the junctions. ${ }^{45}$

A good candidate for the on-site dynamics is a superconducting wire network. In this case one has to take into account the dynamics of the complex order parameter which is given by the time dependent Ginzburg-Landau equation (TDGL) coupled to the electromagnetic field equations. ${ }^{46-48}$ There are two dissipative mechanisms in this case: (i) via the normal state resistivity, since the total current is the sum of the supercurrent and the normal current in each wire of the network (this is the equivalent of the shunt resistance of the RSJ model in a JJA), and (ii) via the relaxation of the complex order parameter in the TDGL equations, which is local in nature ${ }^{48}$ and where its dominant contribution is determined by $D$, the normal state diffusion constant. After writing the TDGL equations in a discrete lattice, and neglecting the fluctuations of the amplitude of the order parameter (London limit), one obtains ${ }^{49}$ that the on-site part in the dynamics of the phase is provided by $D$, and would correspond to Eq. (8) with $\Gamma_{\theta}=16 \pi^{3} D \lambda^{2} /\left(\Phi_{0}^{2} a S\right)$, where $a$ is the network lattice constant and $S$ the section of the wires. Therefore, there is no need to invoke a "leakage of normal current to the ground" in this case. The full dynamics of the superconducting wire network is a mixture of both the "on-site" dynamics and the "RSJ" dynamics. However, in the presence of an on-site contribution, the resulting rate of change of the phases at different sites, like Eq. (8), does not have a logarithmic nonlocal dependence at large separations as in the pure RSJ model. ${ }^{13}$

In any case, in the present work, we will take the purely on-site dynamical equations as a model dynamics that corresponds to a limit of the general dynamics of a JJA or a superconducting wire network where only local dissipation is taken into account. The opposite limit for the dynamics is the pure RSJ model that we have analyzed in Ref. 38.

We integrate the dynamical equations with a second order Runge-Kutta-Helfand-Greenside method with time step $\Delta t$ $=0.01-0.07 \tau$, averaging over, typically, $10^{6}$ time steps after using $5 \times 10^{5}$ time steps for equilibration. The results were averaged over 5-10 different initial configurations of the phases and system sizes ranging from $L=8$ to $L=180$ were considered.

\section{DYNAMIC SCALING THEORY}

Near a second-order phase transition, the diverging correlation length $\xi$ leads to critical slowing down characterized by relaxation times $\tau$ that also diverge approaching the tran- 
sition temperature. The dynamic scaling hypothesis ${ }^{42}$ asserts that measurable quantities should scale with the diverging correlation length $\xi$ and the relaxation time $\tau \propto \xi$, near the transition temperature, where $z$ is the dynamical critical exponent. A general dynamic scaling theory for the resistivity behavior near a superconducting transition has been provided by Fisher, Fisher, and Huse. ${ }^{43}$ According to this scaling theory, the nonlinear resistivity $E / J$ should satisfy the scaling form

$$
T \frac{E}{J}=\xi^{-z} g_{ \pm}\left(\frac{J}{T} \xi\right)
$$

in two dimensions, where the + and - correspond to the behavior above and below the transition, respectively. For a transition in the KT universality class, the correlation length should diverge exponentially as $\xi \propto \exp \left(b /\left|T / T_{c}-1\right|^{1 / 2}\right)$, above $T_{c}$. Otherwise, for a usual continuous transition, a power-law behavior is expected, $\xi \propto\left|T / T_{c}-1\right|^{-\nu}$, with an exponent $\nu$ to be determined. Thus, a scaling plot according to Eq. (16) can be used to verify the dynamic scaling hypothesis and the assumption of an underlying equilibrium transition.

The scaling form of Eq. (16) does not take into account finite-size effects and so it is valid only in a range of temperature $T$ and current densities $J$ where such effects are not dominant. Finite-size effects are very important sufficiently close to the transition when the correlation length $\xi$ reaches the system size $L$. In particular, at $T_{c}$, the correlation length $\xi$ will be cutoff by the system size $L$ in any finite system. From Eq. (16), the nonlinear resistivity at $T_{c}$ should then satisfy the scaling form

$$
T \frac{E}{J}=L^{-z} g\left(\frac{J}{T} L\right) .
$$

It follows from Eq. (17) that the linear resistance $R_{L}$ $=\lim _{J \rightarrow 0} E / J$ should decrease as a power-law of the system size

$$
R_{L} \propto L^{-z}
$$

right at $T_{c}$. This behavior is independent of the form of the correlation length divergence. The linear resistance can be obtained from the Kubo formula of equilibrium voltage fluctuations as

$$
R_{L}=\frac{1}{2 T} \int d t\langle V(t) V(0)\rangle
$$

without an imposing driving current. $R_{L}$ can also be determined more accurately from the long-time fluctuations of the total phase difference across the system $\Delta \theta(t)=L \alpha_{\mu}$ as ${ }^{12,41}$

$$
R_{L}=\frac{1}{2 T t}\left\langle[\Delta \theta(t)-\Delta \theta(0)]^{2}\right\rangle,
$$

valid for sufficiently long times $t$.

The critical dynamics leading to the resistivity scaling described earlier can also be studied by the behavior of time correlation functions. For the frustrated JJA, there are two different types of time correlations of particular interest, the

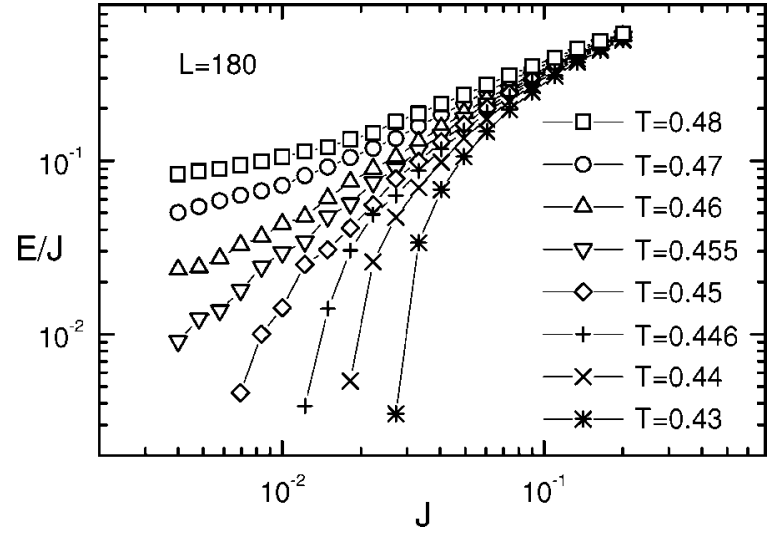

FIG. 1. Nonlinear resistivity $E / J$ as a function of temperature for system size $L=180$.

time correlation for chiralities $C_{c h}(t)$ and phase variables $C_{p h}(t)$. We shall use normalized time correlation functions defined as

$$
C(t)=\frac{\langle A(t) A(0)\rangle-\langle A\rangle^{2}}{\left\langle A^{2}\right\rangle-\langle A\rangle^{2}} .
$$

For the phase variables, $A=\vec{S}=\sum_{i} \vec{s}_{i}$, where $\vec{s}$ $=[\cos (\theta), \sin (\theta)]$ and for the chiral variables $A=\chi=\sum_{\langle i j\rangle}\left(\theta_{i}\right.$ $\left.-\theta_{j}-A_{i j}\right) / 2 \pi$, where the summation is taken over the elementary plaquette of the lattice and the gauge-invariant phase difference is restricted to the interval $[-\pi, \pi]$. The relaxation time $\tau$ can be obtained from the exponential decay $C(t) \propto \exp (-t / \tau)$ at sufficiently long times. In general, the time dependence of $C(t)$ can be expressed as a series of exponential terms with the largest decay time corresponding to the critical relaxation time of the long time dynamics. ${ }^{50}$ From dynamic finite-size scaling, the relaxation time should scale at $T_{c}$ as $\tau \propto L^{z}$, from which the $z$ can be estimated from the slope in a $\log \log$ plot. An alternative procedure to estimate $z$ from equilibrium dynamics is to explore the expected finite-size behavior of the time correlation functions at long times. Since at $T_{c}$ the relaxation time scales as $\tau \propto L^{z}$, the time correlation function for different system sizes can be cast into a scaling form in terms of the dimensionless ratio $t / L^{z}$ as

$$
C(L, t)=\tilde{C}\left(t / L^{z}\right)
$$

where $\widetilde{C}(x)$ is a scaling function. However, this assumes a simple scaling form for the time correlation functions and is only valid for sufficiently long times when a single exponential term describes the relaxation behavior.

\section{RESULTS AND SCALING ANALYSIS}

Figure 1 shows the temperature dependence of the nonlinear resistivity $E / J$ for the largest systems size $L=180$ near the chiral transition temperature $T_{c h}$, estimated previously from equilibrium Monte Carlo simulation, ${ }^{18} \quad T_{c h}=0.455$. Qualitatively, the linear resistance $R_{L}=\lim _{J \rightarrow 0} E / J$, tends to a finite value at high temperatures but extrapolates to very low 


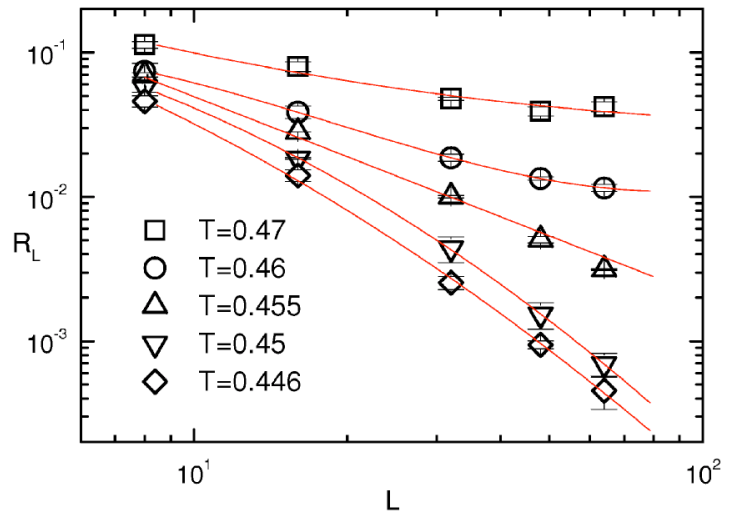

FIG. 2. Linear resistance $R_{L}$, obtained without current bias, as a function of temperature and system size. Lines are just guide to the eyes.

values at lower temperatures, consistent with the existence of a resistive transition in the range $0.45<T_{c}<0.46$. In the double transition scenario, where the phase-coherence transition is expected to be in the KT universality class, the estimate of the proposed $\mathrm{KT}$ critical temperature is $T_{K T}$ $=0.446$, from Monte Carlo simulations, ${ }^{24}$ which is close to $T_{c h}$. However, as it is clear from the behavior at the lowest currents in Fig. 1, this estimate is below the resistive transition since the resistivity curves for $T=T_{K T}=0.446$ and $T$ $=0.45>T_{K T}$ tends to zero for $J \rightarrow 0$, indicating that the system is still in the superconducting phase for these temperatures. On the other hand, the resistivity curve for $T=0.46$ $>T_{c h}$ clearly tends to finite resistivity for $J \rightarrow 0$. This shows that the resistivity transition occurs at $T_{c h}$ or at a temperature very close to $T_{c h}$ rather than at the proposed estimate of $T_{K T}$.

Additional support for a resistivity transition at $T_{c h}$ $=0.455$ is provided by the behavior of the linear resistivity $R_{L}$ as a function of system size, shown in Fig. 2. For $T$ $>0.455, R_{L}$ extrapolates to a finite value consistent with the behavior of the nonlinear resistivity for $J \rightarrow 0$ in Fig. 1. On the other hand, for $T \leqslant 0.455$ it extrapolates to zero, indicating that the resistive transition temperature is compatible with the estimate of $T_{c h}=0.455$. Since in this calculations $R_{L}$ is obtained without any current bias, from the equilibrium dynamical fluctuations, according to Eq. (19), this result also verify that the $T_{c}$ inferred from the behavior of the nonlinear resistivity for the largest system size in Fig. 1 is not an artifact of finite current bias and in fact reflects the underlying equilibrium transition behavior.

Although the resistivity behavior of Figs. 1 and 2 already suggest that the resistive transition temperature coincides with $T_{c h}$ or it is much closer to this value than previous estimates, we now proceed, as in any study of critical phenomena, to obtain the asymptotic equilibrium critical behavior in the thermodynamic limit, $L \rightarrow \infty$ and $J \rightarrow 0$, from a scaling theory. A scaling plot according to Eq. (16) is shown in Fig. 3 for the largest system sizes, in the temperature range closest to $T_{c h}$ and smallest current densities, assuming the correlation length $\xi$ has a power-law divergence with $T_{c}=T_{c h}$ and using $\nu$ and $z$ as adjustable parameters so that the best data collapse is obtained. This scaling plot shows that the two largest system sizes $L=128$ and $L=180$ give the

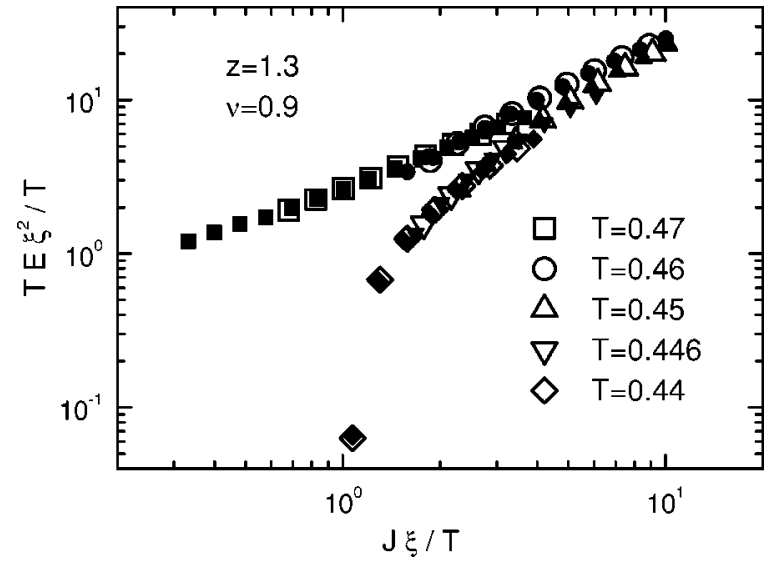

FIG. 3. Scaling plot of the nonlinear resistivity data for the smallest current densities near $T_{c}=T_{c h}=0.455$ with $\xi \propto\left|T / T_{c}-1\right|^{-\nu}$. Open symbols correspond to $L=128$ and filled ones to $L=180$.

same data collapse and so finite size effects neglected in the scaling form of Eq. (16) are not dominant for the range of temperatures and current densities shown in the plot. Similar scaling analysis assuming a KT correlation length and fixing $T_{c}$ at the estimate of $T_{K T}$ does not result in a good data collapse. The same behavior was found using the RSJ dynamics. ${ }^{38}$ From this scaling analysis, we estimate $\nu$ $=0.9(1)$ and the dynamical critical exponent $z=1.3(3)$. The static exponent $\nu$ is consistent with estimates of the chiral transition from equilibrium Monte Carlo simulations ${ }^{18}$ but the accuracy is not sufficient to rule out the value $\nu=1$ expected for the standard Ising transition. Our estimate of $z$ is smaller than the one obtained previously for the frustrated $X Y$ model with MC vortex dynamics ${ }^{35}$ where $z \sim 2$ was found. However, such MC simulation corresponds to a different dynamics and also only very small system sizes (with $L=8-14$ ) were analyzed. We now take into account finitesize effects explicitly by studying the scaling behavior of the linear resistivity $R_{L}$ near $T_{c}$ in Fig. 2. At $T_{c}$, the linear resistivity should scale with system size according to Eq. (18). Near $T_{c}$, it should also depend on temperature through the dimensionless variable $L / \xi$. If the correlation length diverges as a power law then it should satisfy the finite-size scaling form

$$
R_{L} L^{z}=f\left[\left(T / T_{c}-1\right) L^{1 / \nu}\right] .
$$

In fact, as shown in Fig. 4 the linear resistivity data satisfy the scaling form with $T_{c}=T_{c h}$ and a value $z=1.5(2)$ consistent with the estimate from the nonlinear resistivity scaling.

The earlier scaling analysis for the nonlinear resistivity at large system sizes and linear resistivity at smaller system sizes already confirm that the resistive transition temperature $T_{c}$ is very close to $T_{c h}$, with a dynamic exponent $z<2$. However, in the absence of a completely satisfactorily determination of $T_{c}$ from static critical behavior, ${ }^{20,21,24}$ from now on, we will assume $T_{c}=T_{c h}$ and explore to which extent this give us consistent results for the dynamical critical behavior, including finite-size effects. Another reason to assume the value of $T_{c}$ obtained from equilibrium simulations rather than estimating from the dynamic scaling itself is that, in 


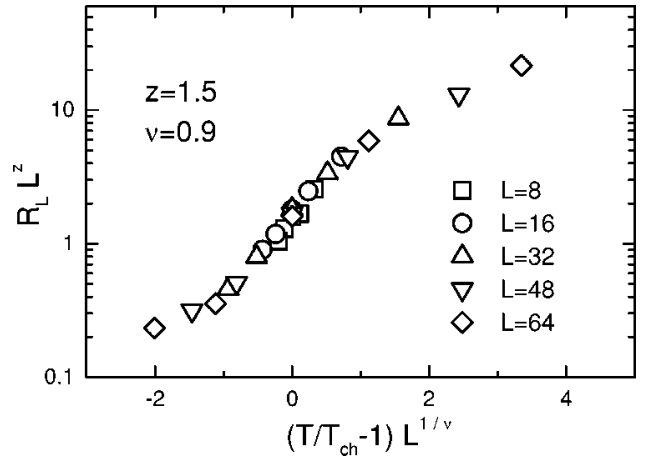

FIG. 4. Finite-size scaling plot of the linear resistance data near $T_{c}=T_{c h}=0.455$.

general, the most reliable way of studying critical dynamics and determine the dynamic exponent $z$ is to use the known value of $T_{c}$. This is true not only for models where $T_{c}$ is known exactly as for the two-dimensional Ising model ${ }^{51}$ but also for models where $T_{c}$ is only known by numerical simulations as for the three-dimensional Ising model. ${ }^{50}$

An alternative estimate of $z$ can be obtained from the nonlinear resistivity by studying the expected size dependence at $T_{c}$. As shown in Fig. 5, a finite size scaling according to Eq. (17) gives the same dynamic exponent $z=1.4(3)$, within the estimated error bar. The same behavior was also observed using the RSJ dynamics ${ }^{38}$ but with a smaller value of $z$. Equilibrium calculations of the linear resistance $R_{L}$ at $T_{c h}$ also give a consistent estimate. Figure 6 shows the finite size behavior of $R_{L}$ obtained from Eq. (18). A power-law fit gives $z=1.41(5)$ which agrees with the other estimates and suggests therefore that the value of $z$ corresponds to the underlying equilibrium dynamical behavior. To show the reliability of this method, it is also included in Fig. 6 the behavior for the unfrustrated case, $f=0$. In this case the resistive transition is in the KT universality class and a dynamical exponent $z=2$ is expected, independent of the dynamics. Indeed, for $f=0$, the same power-law fit at the critical temperature $T_{c}=0.887$ estimated from Monte Carlo simulations ${ }^{52}$ gives $z=1.96(5)$, in good agreement with previous resistivity calculations $^{12}$ for $f=0$ using smaller system sizes up to $L$ $=16$.

It should be noted that our earlier estimate of the dynamic exponent $z$ is obtained by requiring that $T_{c}, z$, and $\nu$ satisfy at the same time the finite-size scaling forms of Eqs. (17), (18),

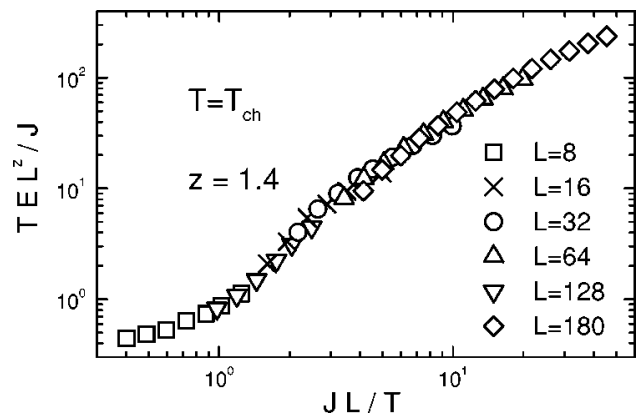

FIG. 5. Scaling plot of the nonlinear resistivity $E / J$ at $T_{c}=T_{c h}$ $=0.455$ for different system sizes $L$.

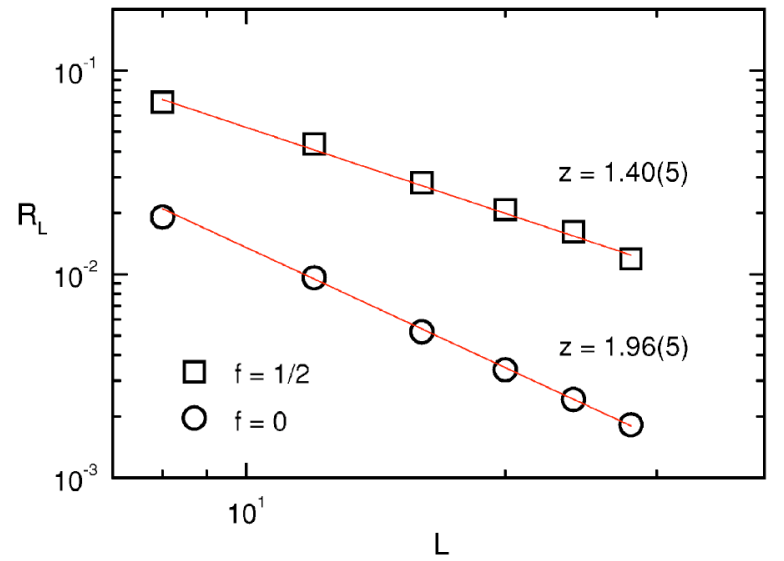

FIG. 6. Linear resistance as a function of system size at the critical temperatures $T_{c}=T_{c h}$ for $f=1 / 2$ and $T_{c}=0.887$ for $f=0$. Power-law fits give estimates of the dynamic exponent $z$.

and (23), including small system sizes, as well as the scaling form of Eq. (16) for the largest system sizes. Using only Eq. (16) can lead to inaccurate estimates of $z$ as shown recently in Ref. 53 for the unfrustrated case.

To further verify that the estimate of $z$ obtained from the resistivity scaling does in fact reflect critical phase fluctuations near the transition rather than just critical fluctuations for the chiral order parameter, we have also performed equilibrium calculations of the phase autocorrelation functions $C_{p h}(t)$ for the phase variables and $C_{c h}(t)$ for the chirality variables. Figures 7 and 8 show the finite-size behavior of the time correlations functions evaluated at the critical temperature $T_{c h}$. If this temperature corresponds to the critical point for phase coherence and vortex-lattice disorder then the relaxation times for both phase and chirality variables should diverge with the system size as $\tau \propto L^{z}$. The relaxation time $\tau_{p h}$ and $\tau_{c h}$ can be obtained from the exponential decay of $C(t)$ at sufficiently long times. We take into account possible contributions from short time behavior by fitting the time dependence of $C_{p h}(t)$ and $C_{c h}(t)$ to a sum of two exponentials and extract $\tau$ from the largest decay time. Figure 9 shows the finite-size behavior of the relaxation time at $T_{c h}$ for the phases and chiralities. From a power-law fit we obtain $z_{p h}$

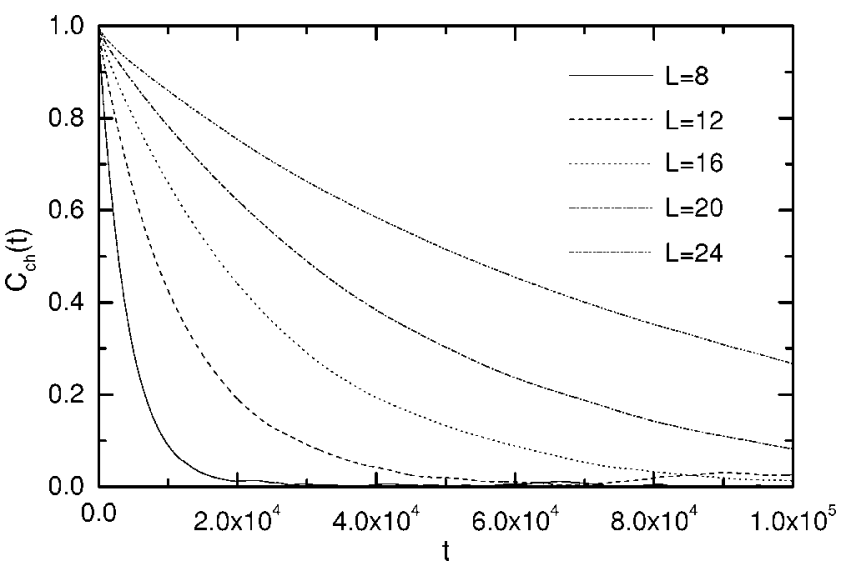

FIG. 7. Time correlation function $C_{c h}(t)$ for the chiral variables at $T_{c h}$, for different system sizes. 


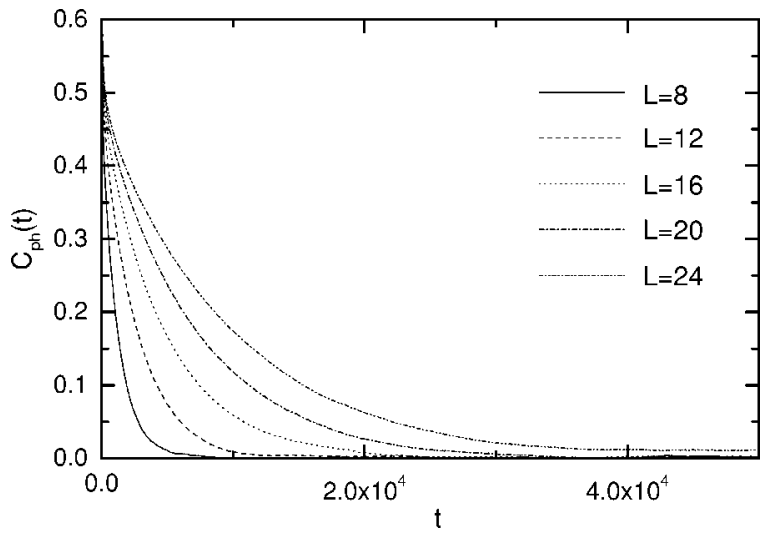

FIG. 8. Time correlation function $C_{p h}(t)$ for the phase variables at $T_{c h}$, for different system sizes.

$=1.8(1)$ from the phase relaxation time $\tau_{p h}$ which is indeed consistent, within the estimated error bar, with the value of $z$ obtained from the resistivity scaling discussed earlier. The estimate from the chiral relaxation time in Fig. 9 is significantly different, $z_{c h}=2.5(2)$. For an alternative estimate of $z$ we have also used the scaling of the correlation function itself. The time correlation functions should satisfy the scaling behavior of Eq. (22). As shown in Figs. 10 and 11, $C_{p h}(t)$ and $C_{c h}(t)$ indeed satisfy the expected finite-size behavior at the critical temperature providing additional estimates of the dynamic exponents $z_{p h}=1.9(2)$ and $z_{c h}=2.6(2)$ which are consistent, within the estimated error bar, with the values obtained from the relaxation time scaling. Finally, above $T_{c}$, the relaxation time should depend both on system size and temperature. If the correlation length diverges as a power law then $\tau_{p h}$ and $\tau_{c h}$ should satisfy the finite-size scaling form

$$
\tau L^{-z}=f\left[\left(T / T_{c}-1\right) L^{1 / \nu}\right] .
$$

In fact, the data collapse in Figs. 12 and 13 show that this scaling form is satisfied with $T_{c}=T_{c h}$ and the values of $\tau_{p h}$ and $\tau_{c h}$ which are consistent with the above estimates.

\section{DISCUSSION}

Recently, Holzer et al. ${ }^{53}$ showed that for the unfrustrated case, $f=0$, the scaling behavior in Eq. (16) considered alone, i.e., without taking into account finite-size effects, yields in-

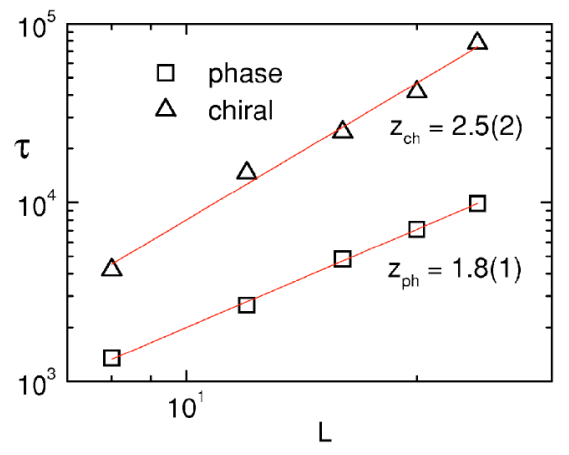

FIG. 9. Finite-size behavior of the phase and chiral relaxation times, $\tau_{p h}$ and $\tau_{c h}$, respectively, at the critical temperature $T_{c}=T_{c h}$. Power-law fits give estimates of the dynamical exponents $z_{p h}$ and $z_{c h}$

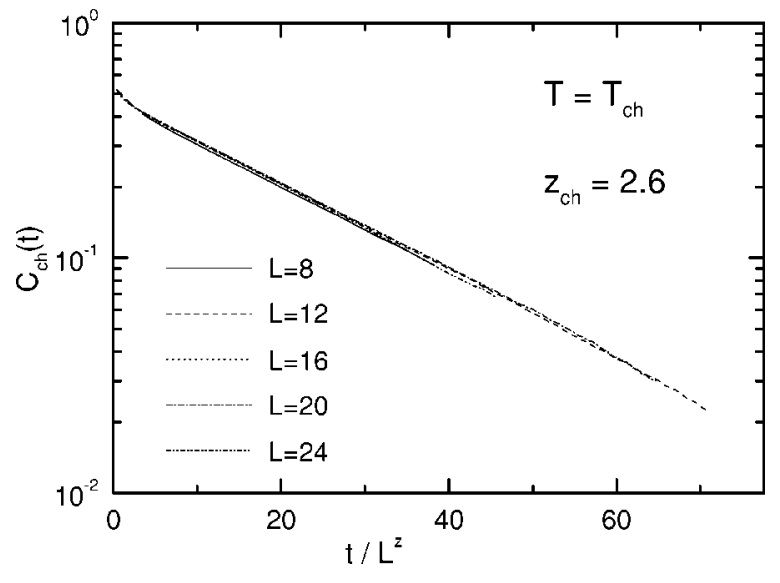

FIG. 10. Finite-size scaling plot of the time correlation function $C_{c h}(t)$.

correct values for the dynamic exponent $z$, using approximate analytical expressions for the $I-V$ characteristics available in the literature. ${ }^{1}$ We should emphasize that our approach for the resistivity scaling analysis described in the previous section is quite different. Our estimate of the dynamic exponent $z$ is obtained by requiring that $T_{c}, z$, and $\nu$ satisfy at the same time the finite-size scaling forms of Eqs. (17), (18), and (23), including small system sizes, as well as the scaling form of Eq. (16) for the largest system sizes. It should also be considered that the possibility of an equilibrium $\mathrm{KT}$ transition for $f=1 / 2$ within the separated transitions scenario does not imply that the dynamics would be the same as the KT dynamics and therefore for the frustrated case considered here there is no reliable analytical expressions available for the $I-V$ characteristics. The dynamics for $f$ $=1 / 2$ will be different because besides vortex excitations, chiral domain walls also contribute to the nonlinear resistivity as shown in Ref. 33. Moreover, it has already been shown for the $f=0$ case that, when finite-size scaling is taking into account in the resistivity scaling theory of Fisher et al., ${ }^{43}$ as we also do in our approach, the correct dynamic exponent $z=2$ is obtained for the KT transition, as shown for example in Ref. 12. This is also verified in the scaling analysis of our data as shown in Fig. 6, where we find a dynamic exponent consistent with $z=2$ for $f=0$, as expected.

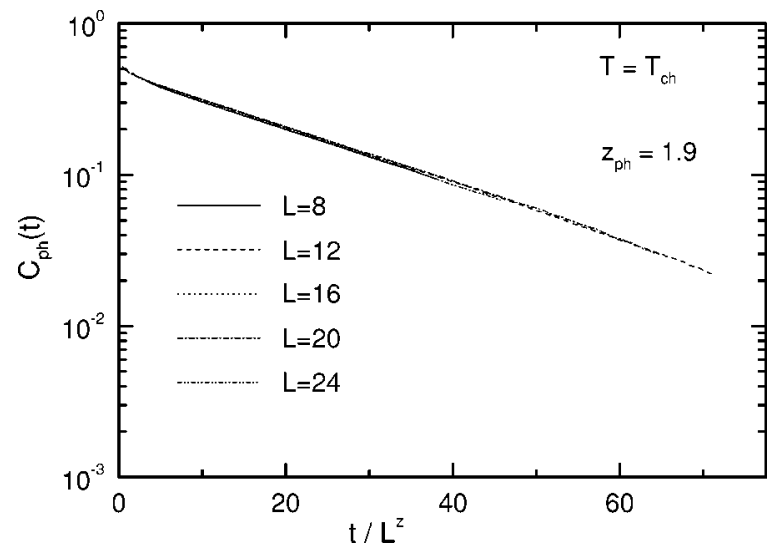

FIG. 11. Finite-size scaling plot of the time correlation function $C_{p h}(t)$. 


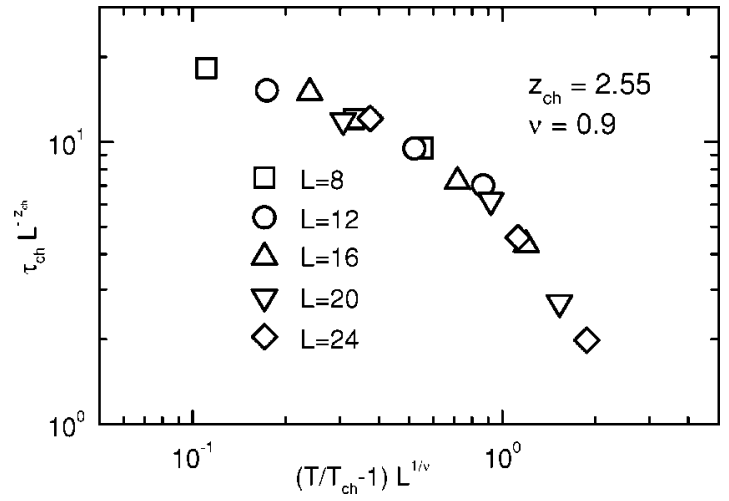

FIG. 12. Finite-size scaling plot for the relaxation time $\tau_{c h}$.

The distinct values obtained for $z_{p h}$ and $z_{c h}$ with the onsite dissipation model deserve some considerations. Similar behavior was also found by us using the RSJ dynamics. ${ }^{38}$ The final results for both models, obtained from the resistivity scaling and time-correlation function scaling analysis, are summarized in Table I. Although for the on-site model, the two methods give results for $z_{p h}$ which differ beyond the estimated error bar, the values are significantly below the value obtained for $z_{c h}$. Naively, if the two transitions happen at the same temperature, one would expect that the same dynamic exponent should hold for the phase and chiral relaxation times. However, we should mention that different dynamic exponents for coupled order parameters have already been found previously at multicritical points in magnetic systems. ${ }^{54}$ This suggests that a possible explanation for two dynamic exponents at the transition of the FJJA may rely on the existence of a multicritical point in the phase diagram of the relevant effective Ginzburg-Landau free energy describing the transition. A multicritical point is known to occur in the coupled $X Y$-Ising model ${ }^{18}$ which should describe the static critical behavior of the FJJA and this could be a useful framework for investigations of the dynamical universality class of FJJA. In the context of superconducting systems, different dynamic exponents for the resistivity and chirality have also recently been found in the resistive transition of disordered superconductors ${ }^{55}$ described by the three-dimensional $X Y$ spin glass model.56 Just as in the case of the frustrated JJA, the phase transition in the $X Y$ spin glass

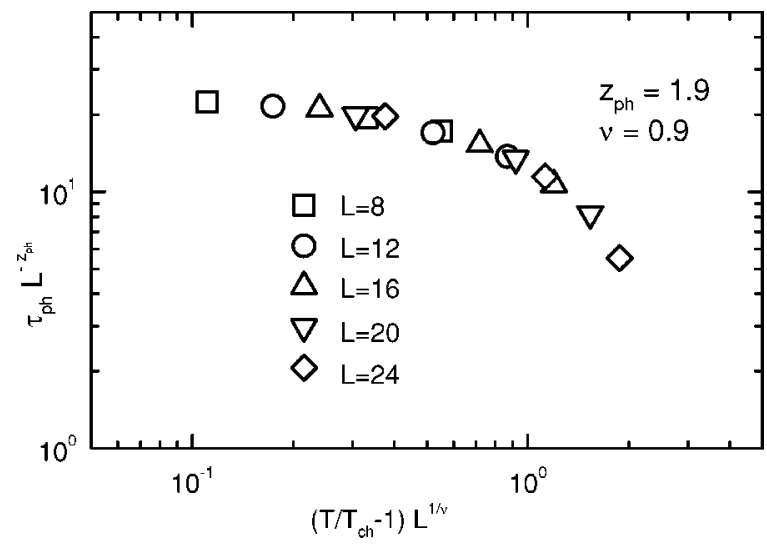

FIG. 13. Finite-size scaling plot for the relaxation time $\tau_{p h}$.
TABLE I. Dynamic exponents of the resistive chiral transition at $T_{c h}$ using the on-site dissipation model (TDGL) and resistivelyshunted-junction model (RSJ). The superscripts $R$ and $C$ correspond to results obtained from the resistivity scaling and time-correlation function scaling, respectively.

\begin{tabular}{lcc}
\hline \hline & RSJ & TDGL \\
\hline$z_{p h}$ & $1.1(1)^{C}, 0.9(1)^{R}$ & $1.8(1)^{C}, 1.4(1)^{R}$ \\
$z_{c h}$ & $2.1(1)^{C}$ & $2.5(2)^{C}$ \\
\hline \hline
\end{tabular}

results from the competition of a chiral order parameter and phase variables. Although earlier work for this problem concluded for a spin-chirality decoupling picture, ${ }^{56}$ more recent numerical work have provided strong evidence ${ }^{57}$ that there is a single transition at which both phase variables and chiralities order.

Although the single transition scenario provides a consistent interpretation of our data, it is worth emphasizing that the alternative separated transitions scenario $^{28}$ cannot be ruled out. We believe, there are two possible explanations for some of our findings within the later scenario, as discussed below.

It is possible that the KT transition is actually much closer to $T_{c h}$ than estimated previously and so the transitions cannot be resolved within the accuracy of our data. Our analysis of the resistivity behavior suggests that in this case it should occur above $T_{c} \sim 0.452$. This value is already close or within the range of the error bars reported for the chiral transition critical temperature obtained, for example, by Monte Carlo simulations which gives $T_{c h}=0.455$ (2) (Ref. 19) or 0.454(2) (Ref. 23). It should be noted, however, that this only considers the critical temperatures alone and not the critical behavior. In the alternative decoupled single transition scenario, the critical behavior should be described by a superposition of a pure KT and pure Ising transitions at the same critical temperature. However, this is also not consistent with our results. Nevertheless, even if the transitions are so close that their critical temperatures can not be resolved by any method, in principle, it could still be possible to distinguish these scenarios due to the mechanism discussed in Ref. 28 or due to the effects of different corrections to scaling.

A second possibility is that the dynamic scaling theory of Fisher et $a l^{43}$ in its original form in Eq. (16) is not valid for the present case and should be enlarged to include the interplay of two divergent length scales at nearby temperatures ${ }^{24}$ which can lead to crossover effects at small length and time scales. In fact, the underlying assumption in the resistivity scaling theory is that there is a single divergent length scale, corresponding to the leading divergent contribution to finite correlation lengths, when approaching the critical temperature of the resistive transition. This would certainly be valid within the coupled single transition scenario, which is consistent with our conclusions since in that case phase coherence and chiral order develop at the same critical temperature, with strongly coupled order parameters, and the equilibrium critical behavior should be described by a single divergent length scale. Above the transition, in the disordered phase, the chiral and phase correlation lengths diverge when 
approaching $T_{c}$ with a common leading divergent contribution. Below the transition, where there is chiral order and a Gaussian fixed line is expected for the phase variables, the chiral correlation length diverges when approaching $T_{c}$ with the same leading divergent contribution while the phase correlation length remains infinite since the Gaussian fixed line corresponds to the absence of a length scale. However, if phase coherence and chiral order develop at different temperatures then the resistivity scaling can only hold sufficiently close to the phase coherence transition otherwise the scaling form of Eq. (16) should be enlarged to include the divergent chiral correlation length in addition to the phase correlation length. This would lead to a scaling function $g_{ \pm}(x, y)$ in Eq. (16) depending on two scaling variables $x$ $=J \xi_{K T} / T$ and $y=\xi_{c h} / \xi_{K T}$, which makes the scaling analysis of the data very complicated specially when taking into account finite-size effects. This could explain, for example, why a good scaling collapse like Fig. 5 is not obtained by assuming a resistive transition at $T_{c}=T_{K T}$, estimated by previous works. However, it would remain unclear to us in this case why the linear and nonlinear resistivity scaling as well as the critical dynamics including different temperatures and system sizes are so well described by a resistive transition at $T_{c}=T_{c h}$.

\section{CONCLUSIONS}

We have studied the resistivity scaling and critical dynamics of a frustrated Josephson-junction array, at $f=1 / 2$ flux quantum per plaquette, by numerical simulations of an onsite dissipation model for the array dynamics. Using a dynamic scaling analysis, we find that the resistivity behavior and critical dynamics are well described by the critical temperature corresponding to the chiral (vortex-lattice) transition with a correlation length that diverges as a power law. Two dynamic exponents, $z_{p h} \sim 1.5$ and $z_{c h} \sim 2.5$, are found for phase coherence and chiral order, respectively. Consequently, at the transition, the exponent of the current-voltage power law, $V-I^{a}$, is $a=z_{p h}+1 \approx 2.5$ rather than $a=3$ as for the unfrustrated case. The same behavior has been found recently for the resistively shunted-junction model ${ }^{38}$ but with different values for the dynamic exponents $\left[z_{p h} \sim 0.9(1)\right.$ and $z_{c h}$ $\sim 2.1]$. One implication of these results for transport experiments is that the usual method of locating the critical temperature from the value corresponding to a nonlinear $I-V$ exponent $a=3$, may lead to a significant underestimate. This is more severe for tunnel-junction arrays which should be better described by the resistively shunted-junction model, ${ }^{13}$ where we expect $a \sim 2$ at the resistive transition. ${ }^{58}$ For wire networks the on-site dissipation model should be more appropriate. Indeed, resistivity scaling of experimental data on wire networks ${ }^{8}$ find $z \sim 2$, which is consistent with our estimate of $z_{p h}$ within errors. It also shows that the resistivity scaling is well described by a power-law correlation length as found in our simulations. Further detailed $I-V$ measurements combined with magnetic properties, which could in principle probe the chiral transition, are needed to test our results.

\section{ACKNOWLEDGMENTS}

This work was supported by a joint grant $\mathrm{CNPq} /$ ProsulBrasil (No. 490096/03-4) and in part by FAPESP (Grant No. 03/00541-0) (E.G.) and CONICET, CNEA, and ANPCyT (PICT99 03-06343) (D.D.).
${ }^{1}$ R. S. Newrock, C. J. Lobb, U. Geigenmuller, and M. Octavio, Solid State Phys. 54, 263 (2000).

${ }^{2}$ B. J. van Wees, H. S. J. van der Zant, and J. E. Mooij, Phys. Rev. B 35, 7291 (1987); H. S. J. van der Zant, H. A. Rijken, and J. E. Mooij, J. Low Temp. Phys. 82, 67 (1991).

${ }^{3}$ J. P. Carini, Phys. Rev. B 38, 63 (1988); R. K. Brown and J. C. Garland, Phys. Rev. B 33, 7827 (1986).

${ }^{4} \mathrm{Ph}$. Lerch, Ch. Leemann, R. Theron, and P. Martinoli, Phys. Rev. B 41, 11579 (1990).

${ }^{5}$ R. Théron, J. B. Simond, Ch. Leemann, H. Beck, P. Martinoli, and P. Minnhagen, Phys. Rev. Lett. 71, 1246 (1993).

${ }^{6}$ Y. J. Yung, I. C. Baek, and M. Y. Choi, Phys. Rev. Lett. 89, 037004 (2002); I. C. Baek, Y. J. Yung, and M. Y. Choi, Phys. Rev. B 69, 172501 (2004).

${ }^{7}$ F. Yu, N. E. Israeloff, A. M. Goldman, and R. Bojko, Phys. Rev. Lett. 68, 2535 (1992).

${ }^{8}$ X. S. Ling, H. J. Lezec, M. J. Higgins, J. S. Tsai, J. Fujita, H. Numata, Y. Nakamura, Y. Ochiai, Chao Tang, P. M. Chaikin, and S. Bhattacharya, Phys. Rev. Lett. 76, 2989 (1996).

${ }^{9}$ H. Beck and D. Ariosa, Solid State Commun. 80, 657 (1991); H. Beck, Phys. Rev. B 49, 6153 (1994); S. E. Korshunov, Phys. Rev. B 50, 13616 (1994).

${ }^{10}$ P. H. E. Tiesinga, T. J. Hagenaars, J. E. van Himbergen, and J. V. José, Phys. Rev. Lett. 78, 519 (1997).
${ }^{11}$ B. J. Kim, P. Minnhagen, and P. Olsson, Phys. Rev. B 59, 11506 (1999).

${ }^{12}$ L. M. Jensen, B. J. Kim, and P. Minnhagen, Phys. Rev. B 61, 15412 (2000).

${ }^{13}$ S. R. Shenoy, J. Phys. C 18, 5163 (1985).

${ }^{14}$ P. Minnhagen, Rev. Mod. Phys. 59, 1001 (1987).

${ }^{15}$ H. Weber, M. Wallin, and H. J. Jensen, Phys. Rev. B 53, 8566 (1996).

${ }^{16}$ (a) S. Teitel and C. Jayaprakash, Phys. Rev. Lett. 51, 1999 (1983); (b) Phys. Rev. B 27, 598 (1983).

${ }^{17}$ M. Y. Choi and S. Doniach, Phys. Rev. B 31, 4516 (1985); M. Yosefin and E. Domany, Phys. Rev. B 32, 1778 (1985); E. Granato and J. M. Kosterlitz, Phys. Rev. B 33, 4767 (1986).

${ }^{18}$ E. Granato, J. M. Kosterlitz, J. Lee, and M. P. Nightingale, Phys. Rev. Lett. 66, 1090 (1991).

${ }^{19}$ J. Lee, J. M. Kosterlitz, and E. Granato, Phys. Rev. B 43, 11531 (1991).

${ }^{20}$ G. Ramirez-Santiago and V. José, Phys. Rev. Lett. 68, 1224 (1992); 77, 4849 (1996).

${ }^{21}$ E. Granato, J. J. Kosterlitz, and M. P. Nightingale, Physica B 222, 266 (1996)

${ }^{22}$ Y. M. M. Knops, B. Nienhuis, H. J. F. Knops, and H. W. J. Blöte, Phys. Rev. B 50, 1061 (1994).

${ }^{23}$ S. Lee and K. C. Lee, Phys. Rev. B 49, 15184 (1994). 
${ }^{24}$ P. Olsson, Phys. Rev. Lett. 75, 2758 (1995); 77, 4850 (1996); Phys. Rev. B 55, 3585 (1997).

${ }^{25}$ V. Cataudella and M. Nicodemi, Physica A 233, 293 (1996).

${ }^{26}$ Y. Honda and T. Horiguchi, Phys. Rev. B 58, 11501 (1998).

${ }^{27}$ E. H. Boubcheur and H. T. Diep, Phys. Rev. B 58, 5163 (1998).

${ }^{28}$ S. E. Korshunov, Phys. Rev. Lett. 88, 167007 (2002).

${ }^{29}$ H. Eikmans, J. E. van Himbergen, H. J. F. Knops, and J. M. Thijssen, Phys. Rev. B 39, 11759 (1989); E. Granato and J. M. Kosterlitz, J. Appl. Phys. 64, 5636 (1988); B. Berge, H. T. Diep, A. Ghazali, and P. Lallemand, Phys. Rev. B 34, 3177 (1986).

${ }^{30}$ The FXY model with modulated couplings has been realized as an array of proximity-effect junctions by varying the junction length [J. Affolter, M. Tesei, H. Pastoriza, Ch. Leemann, and P. Martinoli, Physica C 369, 313 (2002)].

${ }^{31}$ H. J. Luo, L. Schülke, and B. Zheng, Phys. Rev. Lett. 81, 180 (1998).

${ }^{32}$ Y. Ozeki and N. Ito, Phys. Rev. B 68, 054414 (2003).

${ }^{33}$ K. K. Mon and S. Teitel, Phys. Rev. Lett. 62, 673 (1989).

${ }^{34}$ M. V. Simkin, Phys. Rev. B 57, 7899 (1998).

${ }^{35}$ J. R. Lee and S. Teitel, Phys. Rev. B 50, 3149 (1994).

${ }^{36}$ E. Granato, J. M. Kosterlitz, and M. V. Simkin, Phys. Rev. B 57, 3602 (1998); M. V. Simkin and J. M. Kosterlitz, Phys. Rev. B 55, 11646 (1997).

${ }^{37}$ V. I. Marconi and D. Domínguez, Phys. Rev. Lett. 87, 017004 (2001).

${ }^{38}$ E. Granato and D. Domínguez, Europhys. Lett. 63, 750 (2003).

${ }^{39}$ D. Domínguez, Phys. Rev. Lett. 82, 181 (1999); V. I. Marconi and D. Domínguez, Phys. Rev. B 63, 174509 (2001).

${ }^{40}$ B. J. Kim and P. Minnhagen, Phys. Rev. B 60, 588 (1999).

${ }^{41}$ E. Granato, Phys. Rev. B 58, 11161 (1998).

${ }^{42}$ P. C. Hohenberg and B. I. Halperin, Rev. Mod. Phys. 49, 435 (1977).

${ }^{43}$ D. S. Fisher, M. P. A. Fisher, and D. A. Huse, Phys. Rev. B 43, 130 (1991).

${ }^{44}$ P. H. E. Tiesinga, Ph.D. thesis, Utrecht University, 1996.

${ }^{45}$ We acknowledge H. Pastoriza, for suggesting this possibility to us.

${ }^{46}$ (a) A. Schmid, J. Phys.: Condens. Matter 5, 302 (1966); (b) L. P. Gorkov and G. M. Eliashberg, Sov. Phys. JETP 27, 328 (1968); (c) L. Kramer and R. J. Watts-Tobin, Phys. Rev. Lett. 40, 1041 (1978); (d) G. Schon and V. Ambegoakar, Phys. Rev. B 19, 3515 (1979); (e) C. R. Hu and R. S. Thompson, Phys. Rev. B 6,
110 (1972).

${ }^{47}$ A. M. Kadin and A. M. Goldman, in Nonequilibrium Superconductivity, edited by D. N. Langenberg and A. I. Larkin (North Holland, Amsterdam, 1986), p. 253; A. L. de Lozanne and M. P. Beasley, ibid.111; D. Y. Vodolazov, F. M. Peeters, L. Piraux, S. Matefi-Tempfli, and S. Michote, Phys. Rev. Lett. 91, 157001 (2003); S. Michote, S. Matefi-Tempfli, L. Piraux, D. Y. Vodolazov, and F. M. Peeters, Phys. Rev. B 69, 094512 (2004).

${ }^{48}$ The TDGL equations were proposed by Schmid in Ref. 46(a) and were shown rigorously to be valid for gapless superconductors (due to paramagnetic impurities) at all temperatures in Ref. 46(b). More general versions of the TDGL equations, which add a nonlinear term in the time-derivative, were obtained in Ref. 46(c) and 46(d) valid for dirty superconductors near $T_{c}$, including several pair breaking mechanisms, like electron-phonon scattering, paramagnetic impurities, etc. Comparison with experimental results of the TDGL equations (see Ref. 47) in the case of superconducting wires show that the extra nonlinear terms in the time-derivative are important for high currents. In general, there is no universal simple TDGL equation appropriate to all situations in any material. However, in any case, the relaxation of the superconducting order parameter is expected to be always local in space and thus it will provide an on-site dissipation mechanism which is completely different from the pure RSJ model dynamics. In this respect the simple TDGL equations of Ref. 46(a) seem to be enough to describe the asymptotic critical dynamics, as well as the behavior for low currents near the superconducting $T_{c}$.

${ }^{49}$ D. Domínguez and E. Granato (unpublished).

${ }^{50}$ S. Wansleben and D. P. Landau, Phys. Rev. B 43, 6006 (1991).

${ }^{51}$ Martin-D. Lacasse, J. Viñals, and M. Grant, Phys. Rev. B 47, 5646 (1993).

${ }^{52}$ H. Weber and P. Minnhagen, Phys. Rev. B 37, 5986 (1988).

${ }^{53}$ J. Holzer, R. S. Newrock, C. J. Lobb, T. Aouaroun, and S. T. Herbert, Phys. Rev. B 63, 184508 (2001).

${ }^{54}$ D. L. Huber and R. Raghavan, Phys. Rev. B 14, 4068 (1976).

${ }^{55}$ E. Granato, Phys. Rev. B 69, 012503 (2004); 69, 144203 (2004).

${ }^{56}$ H. Kawamura and M. S. Li, Phys. Rev. Lett. 87, 187204 (2001).

${ }^{57}$ L. W. Lee and A. P. Young, Phys. Rev. Lett. 90, 227203 (2003).

${ }^{58}$ The experiments in underdamped JJA of Ref. 2 were interpreted using the KT theory obtaining $a \geqslant 3$, but with $T_{c} \approx 0.3$, which is significantly lower than the theoretical $T_{c}$. 